\title{
Female Hypospadias and Urinary Incontinence: Surgical Solution of a Little-Known Entity
}

\author{
Mario Lima, Niel Di Salvo*, Tommaso Gargano and Giovanni Ruggeri \\ Sant'Orsola Hospital, University of Bologna, Bologna, Italy
}

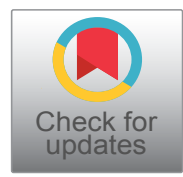

*Corresponding author: Niel Di Salvo, Sant'Orsola Hospital, University of Bologna, Via Massarenti 11, 40138, Bologna, Italy, Tel: +393889066813, E-mail: neildisalvo@hotmail.com

\section{Introduction}

Female hypospadias is a rare genito-urinary anomaly, often associated with other congenital malformations of the urinary tract.

For female hypospadias it is meant a condition in which the external urethral meatus opens high up in the anterior vaginal wall proximal to the hymenal ring; the urethra is obviously shorter than usual.

Embryologically, it is thought to be due to an abnormal development of the urogenital sinus or to a lack of differentiation of Wolff's tissue. Etiology though still remains unknown.

Even though commons symptoms related to female hypospadias include urinary incontinence, recurrent urinary tract infections and dyspareunia, patients are often diagnosed accidentally during catheterization for other reasons.

Its detection is simple but often late in childhood or even in adulthood. Treatment can be difficult due to the lack of consistent literature on the topic and also because it requires management of the other associated anomalies. We describe the case of a 5-year-old girl presenting incontinence secondary to hypospadias, successfully treated with a 2-step surgical strategy.

\section{Presentation of the Case}

We describe the case of a female patient referred to our Institution for urinary incontinence likely due to hypospadias. As a matter of fact, her clinical history was way more complex. She was born at term, with no prenatal diagnostic suspicions. After toilet training achievement, between 2 and 3 years old, her mother noticed a continuous urinary dripping during day and night hours. Simultaneously, she started suffering from well-documented recurrent urinary tract infections (UTIS). She was first evaluated elsewhere with an initial diagnosis of hyperactive bladder, treated with oxybutynin without success until the age of 5 when a Voiding Cystourethrography (VCUG) was performed and diagnosis of II-degree bilateral Vesicoureteral Reflux (VUR) was made. The VUR was then treated endoscopically; during the operative cystoscopy, a female hypospadias was detected. Only at this point, she was referred to our Centre for management of urinary incontinence likely due to female hypospadias. We decided to do a diagnostic work-up from the beginning, starting with ultrasounds that showed us the presence of a right duplex system.

VCUG demonstrated resolution of the previously treated VUR with a lower bladder on the perineal plane, with the base projected constantly under the upper profile of the pubic symphysis; also, a short urethra, of regular calibre, was seen (Figure 1). In fact, over the last years, she had not presented any UTI.

On physical examination under general anaesthesia before cystoscopy, a mega external urethral meatus opening over the superior vaginal wall could be evidently seen (Figure 2).

On the following cystoscopy, besides the subsequent short urethra, an ectopic ureteral orifice under the bladder neck was detected. We also performed a

Citation: Lima M, Di Salvo N, Gargano T, Ruggeri G (2018) Female Hypospadias and Urinary Incontinence: Surgical Solution of a Little-Known Entity. Int Arch Urol Complic 4:049. doi.org/10.23937/2469$5742 / 1510049$

Accepted: November 21, 2018: Published: November 23, 2018

Copyright: (C) 2018 Lima M, et al. This is an open-access article distributed under the terms of the Creative Commons Attribution License, which permits unrestricted use, distribution, and reproduction in any medium, provided the original author and source are credited. 


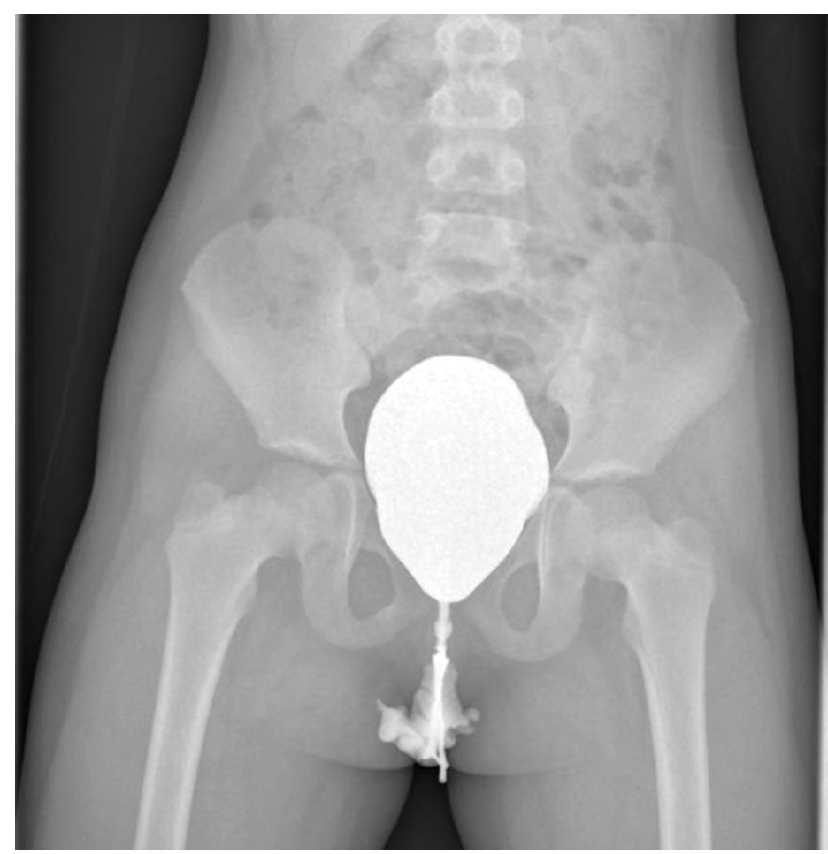

Figure 1: VCUG demonstrates resolution of the previously treated VUR with a lower bladder on the perineal plane, with the base projected constantly under the upper profile of the pubic symphysis; also, a short urethra, of regular calibre, is seen.

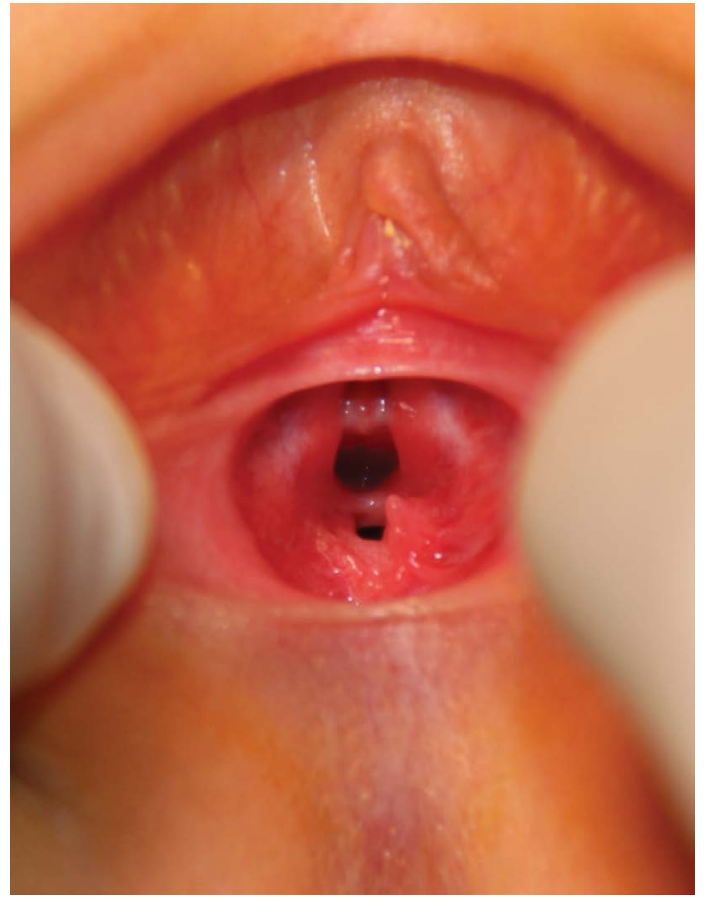

Figure 2: On physical examination, a mega external urethral meatus opening over the superior vaginal wall could be evidently noticed.

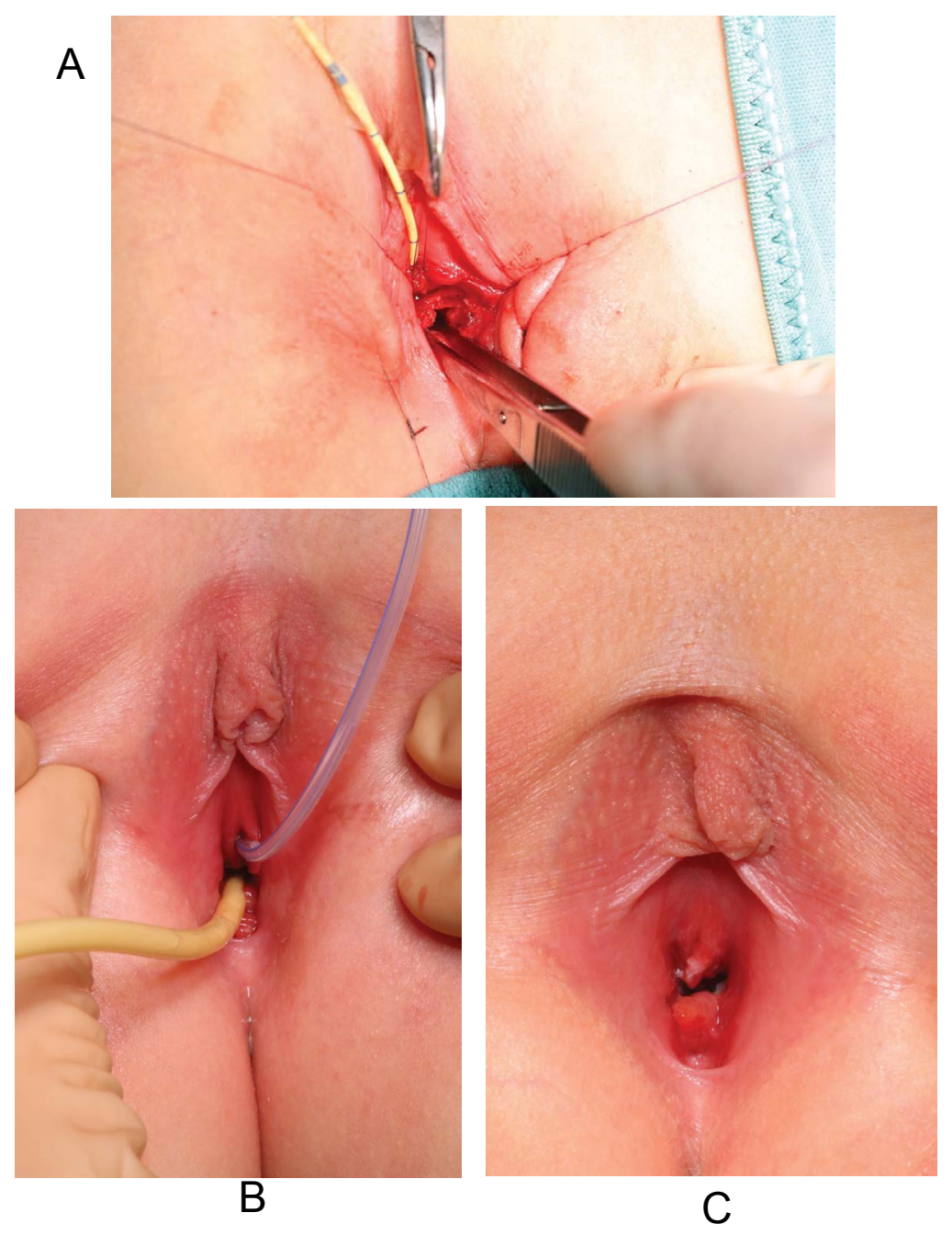

Figure 3: a) Separation of the distal urethra from the superior vaginal wall, both previously incanulated; b) Creation of the 1.5 $\mathrm{cm}$ distal urethra missing in double layers over a guide Foley catheter $\mathrm{Ch} 10 ; \mathrm{c}$ ) The external urethral meatus is now on the vulvar plane. 
urodynamic study. The latter documented a urethralbladder neck incompetence.

At the end of the work-up, this patient's incontinence was both a real incontinence determined by a short urethra on which the perineal muscular complex could not act at best and a pseudo-incontinence determined by ureteral ectopy but also by the hypospadias itself because of vaginal micturition.

At this point, after careful analysis of the limited existent literature on the topic, we planned a $2 / 3$ step program: A distal urethral lengthening through a periurethral vaginal flap urethroplasty in double layer, the ectopic ureter's reimplantation and an eventual bladder neck reconstruction.

We first separated the distal urethra from the superior vaginal wall, both previously incanulated. We then built the $1.5 \mathrm{~cm}$ distal urethra missing in double layers on the guide of a Ch 10 Foley catheter in order to create an external urethral meatus on the vulvar plane (Figure 3a, Figure 3b and Figure 3c). The superior vaginal wall was reconstructed. At the end of the procedure, we put a suprapubic catheter in place in order to protect the neourethra.

The catheter was removed after a month. After the procedure the patient herself reported a substantial improvement in the quantity of involuntary urinary loss, thus proving an important role of female hypospadias regarding incontinence and pseudo-incontinence.

In a second phase, we removed the ectopic ureteral orifice by performing a bladder reimplantation of the right ureters. On the 1-month follow up visit, the patient declared no involuntarily loss of urine. Therefore, the previously planned reconstruction of the bladder neck was no longer necessary.

\section{Discussion}

Female hypospadias is a rare genito-urinary anomaly, often associated with other congenital malformations of the urinary tract. It is generally dealt within the context of urethral malformations that include others, rare as well, such as urethral duplication, urethral stenoatresia, etc... [1].

Female hypospadias without other genito-urinary abnormalities is extremely rare. Only few authors have treated this entity. Its incidence is difficult to assess because many cases are incorrectly classified as urogenital sinus. The consequence of hypospadias not only depends on the site of the meatus but also on the caliber of the urethra. A urethra of normal caliber is more likely to have its meatus close to the introitus. The severity of the hypospadias is variable. A urethra of normal caliber without meatal stenosis may be asymptomatic, coming to light only when an attempt to catheterize the patient is made. It can cause true incontinence, determined by a short urethra on which the pelvic muscular complex could not act at best, but also postmicturition incontinence and imperfect control caused by vaginal voiding (pseudo-incontinence), the urethral syndrome (frequency, dysuria, urgency) and dyspareunia presenting when a sexual active life has started or recurrent UTIs. If the urethra is narrow, the presentation is more likely to have signs of urinary outflow obstruction, ex. distended urinary tract, urinary tract infection, obstructive nephropathy, and hypertension [2]. Embryologically, it is thought to be due to an abnormal development of the urogenital sinus when it is distal, or to a lack of differentiation of Wolff's tissue, when it is proximal [3].

While the majority of male hypospadias may be caused by disruption of androgen signalling during development, the etiology of this condition remains obscure, especially for female hypospadias without other congenital abnormalities. It is interesting that an animal study showed diethylstilbestrol (DES) treatment could induce female hypospadias in mice. Doseresponse analysis indicated that $0.03 \mathrm{~g}$ of DES for 5 days is the lowest known critical dose for hypospadias induction. The authors have shown that DES-induced female hypospadias onset primarily be the result of changes in developing dorsal urethral epithelial cell apoptotic and proliferative activity. The location of DESinduced hypospadias formation is dependent on age at time of exposure $[4,5]$.

The adverse effects of in utero DES exposure in women include tissue and structural anomalies of the reproductive tract like vaginal anomalies; however, to our best knowledge, female hypospadias, in humans, has never been associated to DES exposure. As a matter of fact, our patient did not present any history of prenatal exposure to synthetic nonsteroidal estrogens.

Treatment can be difficult due to the lack of consistent literature on the topic and also because it requires management of the other associated anomalies.

In order to solve the problem of incontinence secondary to hypospadias, surgeons have attempted a mixed variety of bladder neck reconstructive techniques often associated to extensive internal and/or external urethral lengthening $[6,7]$.

The latter, usually represented by a one-stage perineal urethroplasty, increases the urethral and bladder neck resistance, mainly by elongating the urethral segment and folding adjacent muscular and subcutaneous tissue. It is a simple and effective method for development of both continence and cosmesis avoiding initial abdominal surgery that could be reserved for the failure cases. In our case, abdominal surgery was deemed necessary because of the presence of ureteral ectopy.

Our case is in accordance with the experience of Hendren who proposed, in the management of incontinence secondary to female hypospadias, a 
perineal urethral lengthening approach after failure of bladder neck reconstruction alone [7].

\section{References}

1. M Lima, F Destro, M Maffi, D Persichetti Proietti, G Ruggeri (2017) Practical and functional classification of the double urethra: A variable, complex and fascinating malformation observed in 20 patients. J Pediatr Urol 13: 42.e1-42.e7.

2. HM Knight, NJ Philips, PD Mouriquand (1995) Female hypospadias: A case report. J Pediatr Surg 30: 1738-1740.

3. A Chemaou, F Lasry, Z Nejdioui, M Eizmmouri, H Sibai (2013) Female hypospadias diagnosed in an adolescent.
Arch Pediatr 20: 1314-1316.

4. A Bhat, R Saxena, M Bhat, M Dawan, G Saxena (2010) Female hypospadias with vaginal stones: A rare congenital anomaly. J Pediatr Urol 6: 70-74.

5. Miyagawa S, Buchanan DL, Sato T, Ohta $Y$, Nishina $Y$, et al. (2002) Characterization of diethylstilbestrol-induced hypospadias in female mice. Anat Rec 266: 43-50.

6. KA Burbige, TW Hensle (1985) Surgical management of urinary incontinence in girls with congenitally short urethra. J Urol 133: 67-71.

7. WH Hendren WH (1980) Construction of female urethra from vaginal wall and a perineal flap. J Urol 123: 657-664. 